\title{
On the $L_{\infty}$ Walsh Arrays for $\Gamma(x)$ and $\operatorname{Erfc}(x)$
}

\author{
By John R. Rice
}

1. Introduction. Let $f(z)$ be a complex function and let $S$ be a compact subset of the complex plane. We may consider the complex functions to be normed by an $L_{p}$ norm defined either by the surface integral

$$
\|f(z)\|_{p}=\left[\iint_{S}|f(z)|^{p} d z\right]^{1 / p}, \quad p>0
$$

or by the contour integral

$$
\|f(z)\|_{p}=\left[\int_{S}|f(z)|^{p} d z\right]^{1 / p}, \quad \quad p>0 .
$$

In either case it is known [2] that if $f(z)$ is, say, continuous, then there exists a rational function

$$
R_{n, m}(z)=\frac{\sum_{i=0}^{n} a_{i} z^{i}}{\sum_{i=0}^{m} b_{i} z^{i}}
$$

such that

$$
\left\|R_{n, m}(z)-f(z)\right\|_{p}
$$

is a minimum for all rational functions of degree $(n, m)$. Such a rational function is said to be a best $L_{p}$ approximation to $f(x)$. One may form a two-dimensional array of these rational approximations analogous to the Padé table. One has one such an array for a given $f(z)$ and for each combination of $L_{p}$ norm and set $S$. Since best approximations are not always unique, there may be several entries for some $(n, m)$.

$$
\begin{array}{llll}
R_{00}(z) & R_{10}(z) & R_{20}(z) & \ldots \\
R_{01}(z) & R_{11}(z) & R_{21}(z) & \ldots \\
R_{02}(z) & R_{12}(z) & R_{22}(z) & \ldots
\end{array}
$$

We shall call this array the $L_{p}$ Walsh array for $f(z)$ on $S$. These arrays have been studied extensively by J. L. Walsh beginning around 1930 [4] and a large collection of his results on these arrays is contained in [2].

The first row of the $L_{p}$ Walsh array contains polynomials and a very large body of literature exists concerning various properties of this sequence of polynomial approximations. One of the most interesting questions pertaining to these arrays is

Received December 17, 1963. This work was partially supported by Contract Nonr $3722(00)$ of the Office of Naval Research. Reproduction in whole or part is permitted for any purpose of the United States Government. 
the question of the rate of convergence. That is to say, how rapidly do the numbers

$$
E_{n, m}=\left\|f(z)-R_{n, m}(z)\right\|_{p}
$$

tend to zero? For $m=0$, there are many classical results on this question and, more recently, Walsh [3] has obtained some results on behavior of $E_{n, m}$ for any fixed value of $m$ or $n$. Very little is known for other paths in the array.

Each rational function $R_{n, m}(x)$ contains $n+m+1$ parameters or coefficients. A question of considerable interest is: given $p=n+m+1$, determine $n$ and $m$ so that $E_{n, m}$ is minimized. Clearly for each $p$ there is at least one value of $(n, m)$ which satisfies this requirement. This value (or values) are denoted by $E_{n, m}^{*}$ and the corresponding values of $(n, m)$ are denoted by $\left(n^{*}, m^{*}\right)$. The sequence

$$
\left\{\left(n^{*}, m^{*}\right) \mid n^{*}+m^{*}+1=p ; p=1,2, \cdots\right\}
$$

is said to be the path of maximal convergence. The question at the first of this paragraph may be rephrased to: What is the path of maximal convergence?

In this paper we consider two $L_{\infty}$ Walsh arrays. The first is that for the complementary error function $\operatorname{Erfc}(x)$ and the interval $[0, \infty]$. The second is for the Gamma function and the interval [2,3]. The object is to determine an initial segment of the path of maximal convergence for each of these arrays. The determination is made by actual computation of entries of the Walsh arrays.

The entries themselves are not presented here. They will appear in a large collection of approximations to be published in book form.

Thus we will actually consider here the arrays of $E_{n, m}$ associated with the Walsh arrays. There is considerable contrast between the two arrays. The array for $\operatorname{Erfc}(x)$ is very regular and the path of maximal convergence is a smooth curve. One can, with considerable confidence, extrapolate the path of maximal convergence obtained to much larger values of $p$. On the other hand the $L_{\infty}$ Walsh array for $\Gamma(x)$ is highly irregular, as is the initial segment of the path of maximal convergence. The explanation of this irregularity will no doubt require a careful study of the deeper properties of this function. In spite of this irregularity, there is rather good agreement between the results presented here and those obtained by Walsh on the asymptotic rate of convergence to zero of $E_{n, m}$ for $m$ fixed.

2. The Complementary Error Function. The error function is defined here by

$$
\operatorname{Erf}(x)=\frac{2}{\sqrt{\pi}} \int_{0}^{x} e^{-t^{2}} d t, \quad 0 \leqq x \leqq \infty .
$$

Two other forms are commonly used, namely $\operatorname{Erf}(x / \sqrt{2})$ and

$$
\frac{1}{2}[1+\operatorname{sgn}(x) \operatorname{Erf}(|x| / \sqrt{2})] .
$$

The complementary error function is

$$
\operatorname{Erfc}(x)=1-\operatorname{Erf}(x) .
$$

As $x$ become large, Erfc $(x)$ tends to zero very rapidly. We have, for large $x$,

$$
\frac{2 x e^{-x^{2}}}{\sqrt{\pi}\left(2 x^{2}+1\right)} \leqq \operatorname{Erfc}(x) \leqq \frac{e^{-x^{2}}}{x \sqrt{\pi}} .
$$


Let $R_{n, m}(x)$ denote the $(n, m)$ entry in the $L_{\infty}$ Walsh array for the interval $[0, \infty]$. One does not normally consider the Walsh array for infinite intervals, however, in this case, the array is well defined for $n \leqq m$. One would normally be interested in this portion of the array. Let

$$
E_{n, m}^{\prime}=-100 \log \left\|\operatorname{Erfc}(x)-R_{n, m}(x)\right\| \text {. }
$$

A portion of the values of $E_{n, m}^{\prime}$ are given in Fig. 1(a). The largest value of $x$ for which $\left\|\operatorname{Erfc}(x)-R_{n, m}(x)\right\|_{\infty}$ is actually assumed is $x=10.4$ for $R_{3,5}$. Fig. 1(b) shows the initial segment of the path of maximal convergence along with the regular distribution of the sign of $R_{n, m}(0)-\operatorname{Erfc}(0)$. The high degree of smoothness and regularity of the array is illustrated in Fig. 2, where the values of $E_{n, m}$ are plotted for columns of the array. The light lines indicate probable values which have not been computed. The dotted lines are to suggest the smoothness of the "surface" of the $L_{\infty}$ Walsh array for this function. One may ascertain that $E_{n, m}$ tends to zero along the path of maximal degree of convergence at the nearly constant rate. Let $R_{p}{ }^{*}(x)$ denote the best approximation to $\operatorname{Erfc}(x)$ with $p$ parameters, then

$$
\left\|\operatorname{Erfc}(x)-R_{p}{ }^{*}(x)\right\|_{\infty} \sim 1.2(.34)^{p} .
$$

3. The Gamma Function. The Gamma function is defined by

\begin{tabular}{|c|c|c|c|c|c|}
\hline & 0 & 1 & 2 & 3 & 4 \\
\hline 0 & & & & & \\
\hline 1 & 85 & & & & \\
\hline 2 & 133 & 170 & & & \\
\hline 3 & 176 & 226 & & & \\
\hline 4 & 216 & 275 & 309 & & \\
\hline 5 & 255 & 321 & 364 & 390 & \\
\hline 6 & 292 & 365 & 414 & 447 & \\
\hline 7 & & 408 & 462 & 501 & \\
\hline 8 & & 449 & 508 & 552 & 584 \\
\hline 9 & & 489 & 552 & 601 & \\
\hline 0 & & & 597 & 648 & 689 \\
\hline & & & & 693 & \\
\hline
\end{tabular}

$$
\Gamma(x)=\int_{0}^{\infty} t^{x-1} e^{-t} d t
$$

(a)

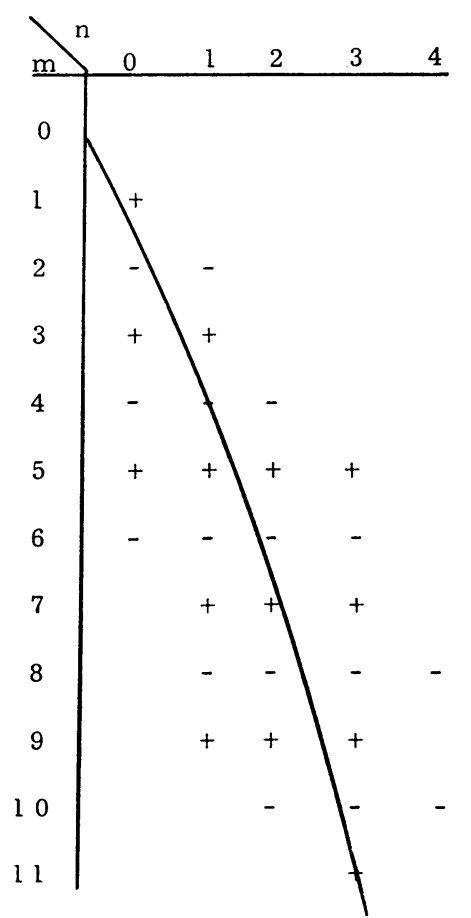

(b)

FIG. 1. (a) The values of $E_{n, m}^{\prime}$ for the initial segment of the $L_{\infty}$ Walsh array of $\operatorname{Erfc}(x)$ on $[0, \infty]$. (b) The signs of $R_{n, m}(0)-\operatorname{Erfc}(0)$ and the initial segment of the path of maximal convergence. 


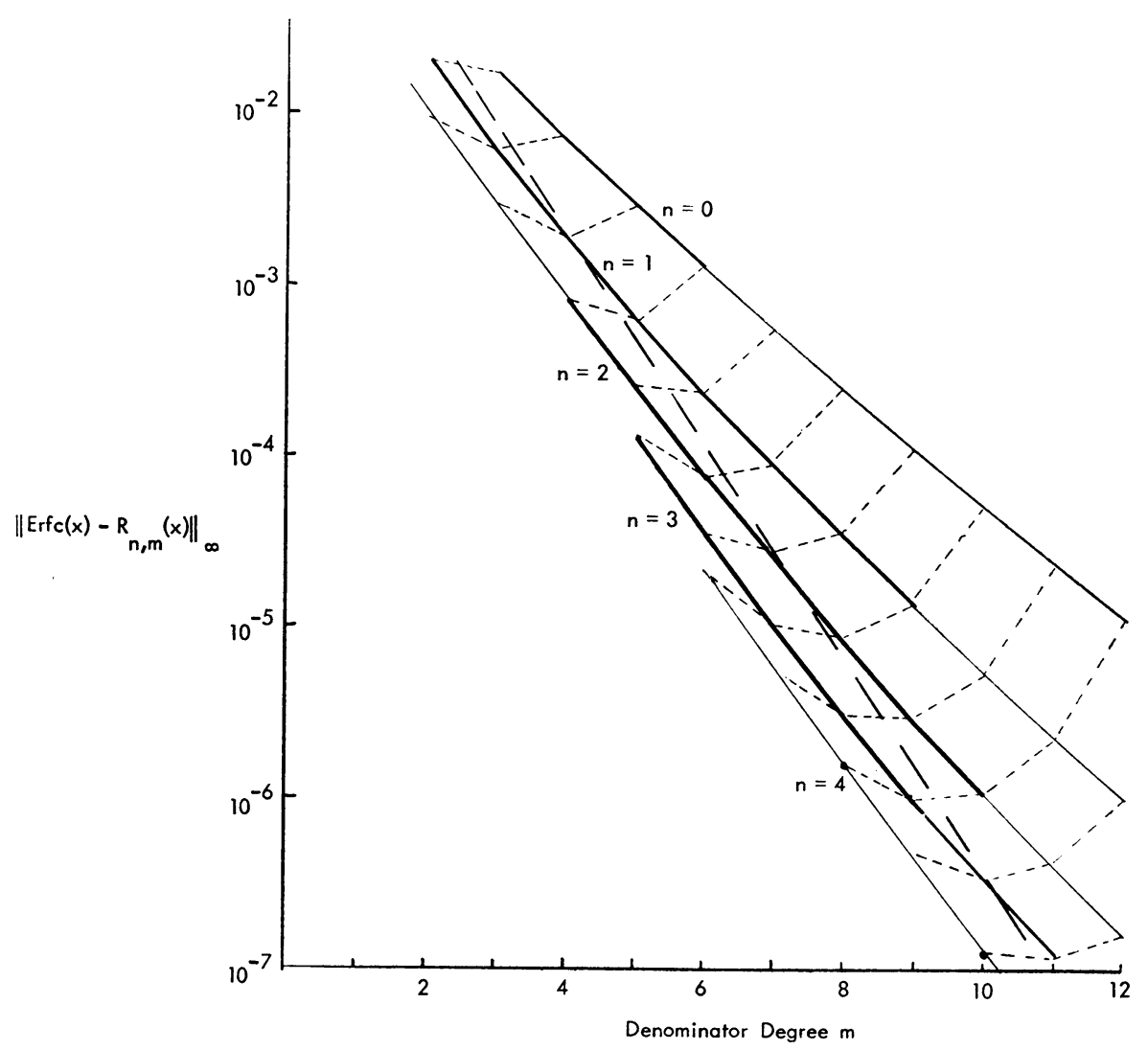
gence.

Fig. 2. The surface of the $L_{\infty}$ Walsh array for $\operatorname{Erfc}(x)$ near the path of maximal conver-

A most notable property of this function is that for an integer $n$,

$$
n !=\Gamma(n+1) \text {. }
$$

The Gamma function increases rapidly with positive $x$, growing as $\sqrt{x} e^{-x} x^{x}$. It has a simple pole at the origin and at each negative integer. This function is difficult to evaluate for small $x$. Apparently, the only feasible method is to use Stirling's formula (or refinements) for large values of $x$ and then use the relation

$$
\Gamma(x)=\frac{\Gamma(x+1)}{x}
$$

to reduce the argument to the desired value.

Again let $R_{n, m}(x)$ denote the $(n, m)$ entry in the $L_{\infty}$ Walsh array for the interval $[2,3]$ and let

$$
E_{n, m}^{\prime}=-100 \log \left\|\Gamma(x)-R_{n, m}(x)\right\|_{\infty} .
$$

A selected number of values of $E_{n, m}^{\prime}$ are given in Fig. 3 . It is not immediately obvious from this table that the "surface" of the Walsh array is highly irregular. This fact becomes more apparent in Fig. 4. There the initial segment of the path 
of maximal convergence is shown along with the sign of $R_{n, m}\left(x_{0}\right)$ where $x_{0}$ is the leftmost extremal point of the error curve $R_{n, m}(x)-\Gamma(x)$. The value of $x_{0}$ is not always 2 . Since not all of the entries in the Walsh array have been computed, it is not certain that the path illustrated is the actual path of maximal convergence. It

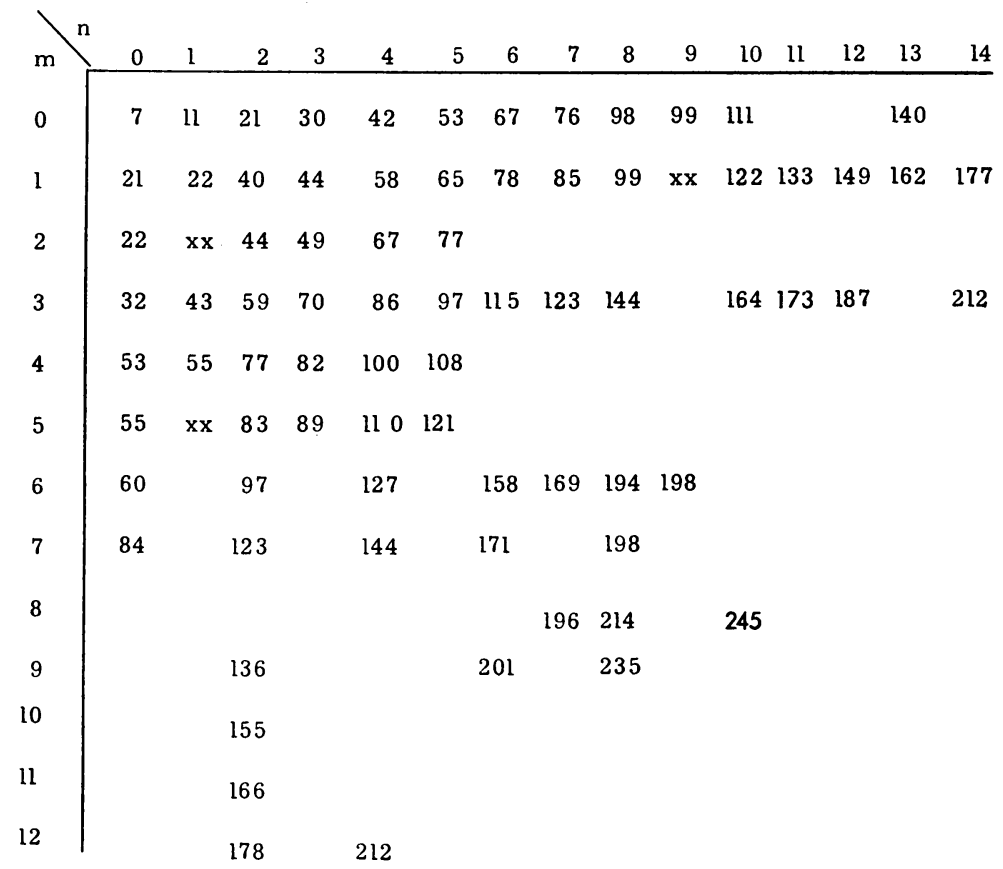

Fig. 3. Selected values of $E_{n, m}^{\prime}$ for the $L_{\infty}$ Walsh array of $\Gamma(x)$ on $[2,3]$. The entries xx indicate entries which require special care in order to be computed.

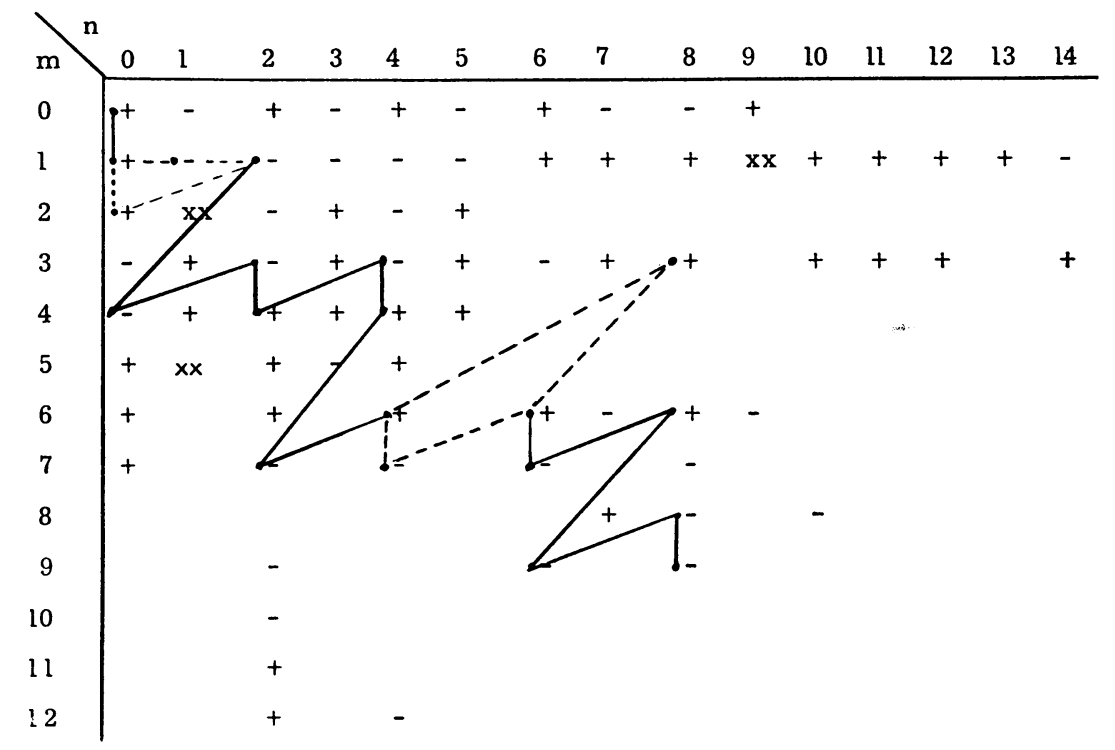

Fig. 4. The initial segment of the path of maximal convergence along with the sign of $R_{n, m}(x)-\Gamma(x)$ at the leftmost extremal point of the error curve. 
is believed that the path shown is correct. The dotted portion of the path corresponds to situations where two entries of the array are equally good approximations.

One might think that entries in Walsh array near the path of maximal degree of convergence would be nearly as good approximations as those on the path. That this is not so is seen from an examination of Fig. 5. In this figure the values of $E_{n, m}^{\prime}$ are plotted with values corresponding to a fixed number of parameters connected by lines. Missing values of $E_{n, m}^{\prime}$ have been estimated to produce a more complete figure. The irregularity of the "surface" of the Walsh array is very apparent in this figure.

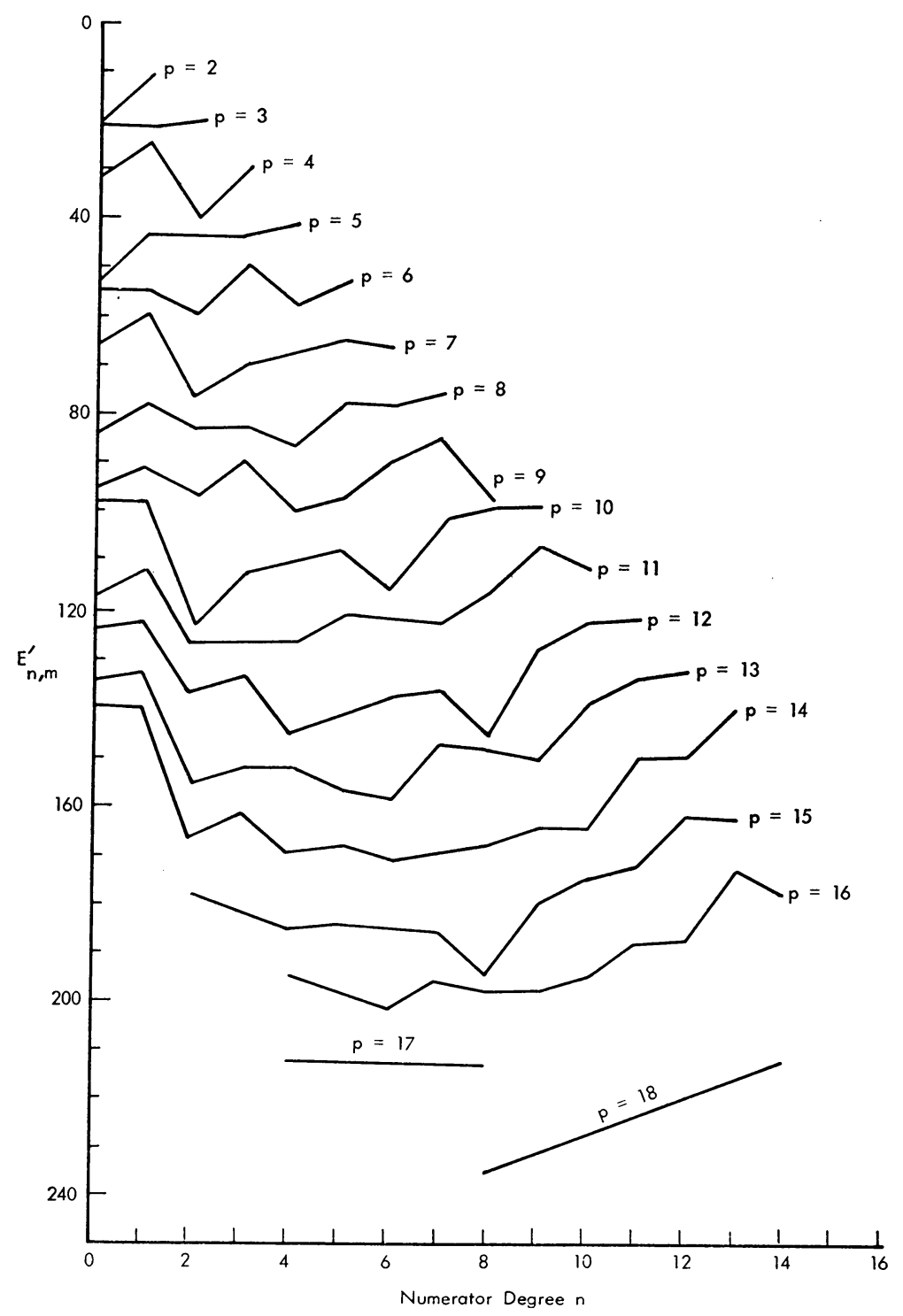

FIG. 5. The values of $E_{n, m}^{\prime}$ joined by lines of $p=n+m+1=$ constant. 
The next two figures show this surface from two other points of view, namely, for lines with constant $n$ and for lines with constant $m$. In these figures, only the known values of $E_{n, m}^{\prime}$ are shown as points connected by solid lines. Conjectured values are not shown, though dotted lines are used to extrapolate and interpolate some of the curves in what is hoped to be a reasonable manner.

In [3] Walsh has obtained estimates for the degree of convergence of $R_{n, m}$ to a given function as $n$ tends to infinity with $m$ fixed. In view of the irregular behavior of the $L_{\infty}$ Walsh array for $\Gamma(x)$, there is surprisingly good agreement between these

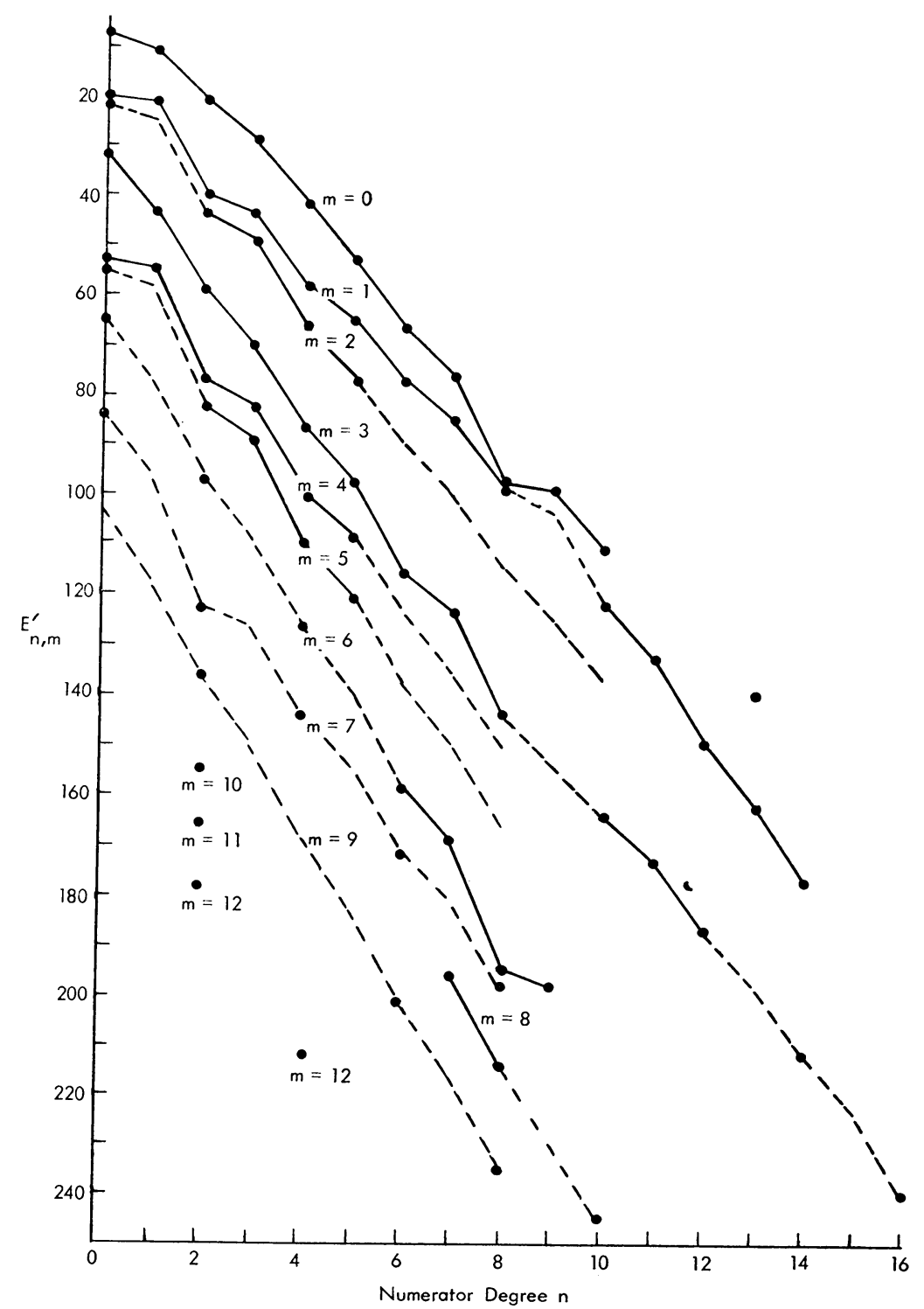

FIG. 6. A view of the surface of the $L_{\infty}$ Walsh array for $\Gamma(x)$ on [2,3]. The lines are for constant degree of the denominator. 
theoretical values and those obtained from Figs. 6 and 7. Walsh's theory states that

$$
\operatorname{Lim}_{n \rightarrow \infty}\left[\left\|\Gamma(x)-R_{n, m}(x)\right\|_{\infty}\right]^{1 / n}=\frac{1}{2 m+5+2 \sqrt{m^{2}+5 m+6}} \sim \frac{1}{4 m+10}
$$

as $n$ tends to infinity. This result and Fig. 6 are compared in Table 1.

Walsh's results also show that the poles of $R_{n, m}(x)$ tend (as $n$ tends to infinity with $m$ fixed) to those $m$ poles of $\Gamma(x)$ closest to the interval [2,3]. This tendency is illustrated in Fig. 8 for $m=1$ and $m=3$.

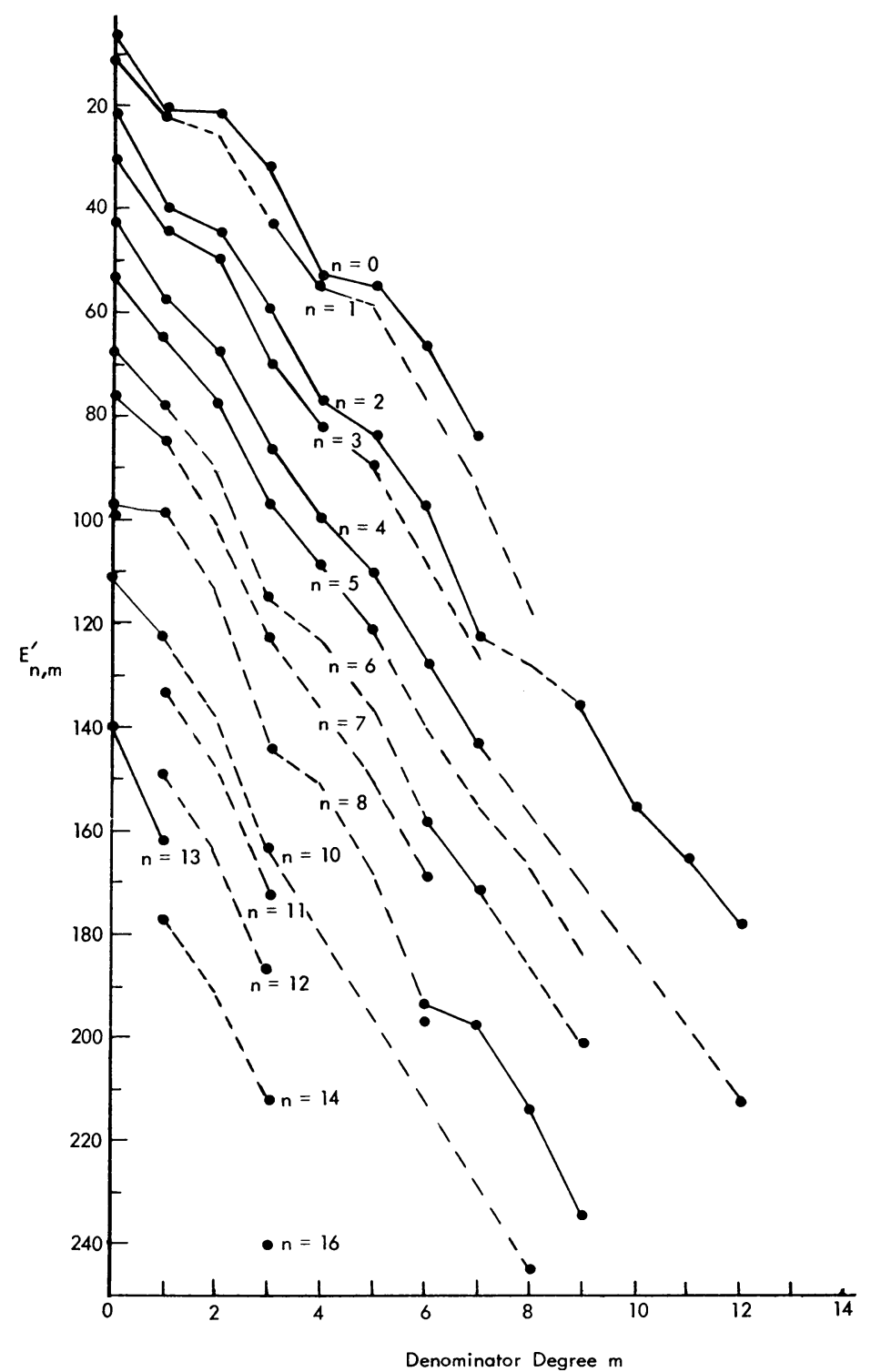

Fig. 7. A view of the surface of the $L_{\infty}$ Walsh array for $\Gamma(x)$ on $[2,3]$. The lines are for constant degree of the numerator. 
TABLE 1

Comparison of the Observed and Theoretical Values of $\left[\left\|\Gamma(x)-R_{n, m}(x)\right\|\right]^{-1 / n}$

\begin{tabular}{c|c|c|c|c|c|c|c|c}
\hline$m$ & 0 & 1 & 2 & 3 & 4 & 5 & 6 & 9 \\
\cline { 2 - 7 } Theory & 10 & 14 & 18 & 22 & 26 & 30 & 34 & 46 \\
Measured & 11.5 & 14 & 17 & 21 & 24.5 & 29.5 & 33 & 47 \\
\hline
\end{tabular}

$m=1$
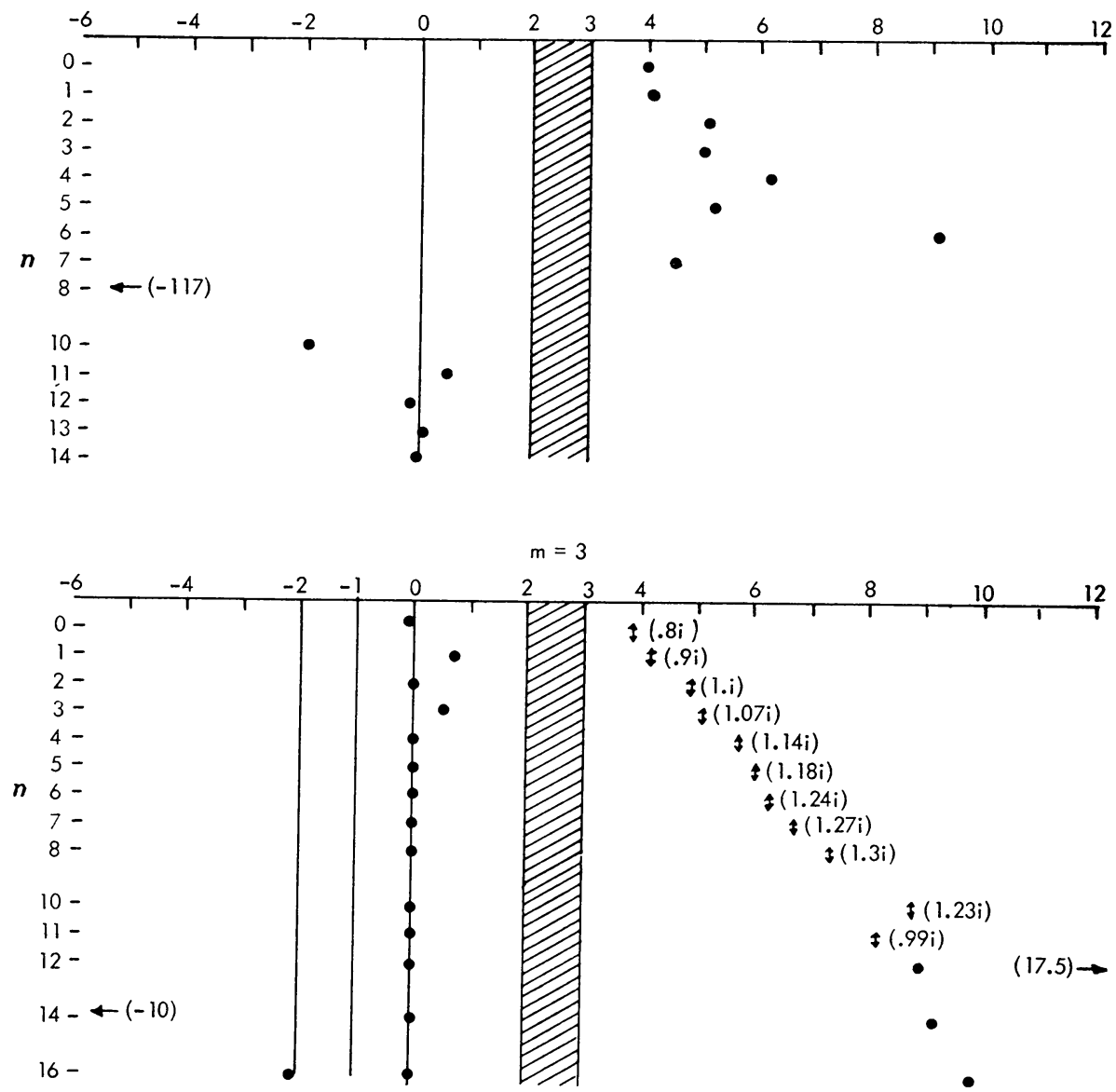

FIG. 8. The location of the poles of $R_{n, 1}(x)$ and $R_{n, 3}(x)$. The real parts of the complex poles are marked by a vertical bar and the imaginary parts are in parentheses.

The irregular nature of the Walsh array for $\Gamma(x)$ is somewhat of a surprise. A foremost open question in this area is the explanation of this irregularity. It seems reasonable that the aberrations in the array are due to the movement of the poles of $R_{n, m}(x)$. Note, for example, that the aberration near $R_{8,0}(x)$ and $R_{9,1}(x)$ corresponds to change of the pole from the right of the interval of $[2,3]$ to the left of it. The pole of $R_{9,1}(x)$ is conjectured to lie very close to 2.0 and its presence there has so far prevented the calculation of $R_{9,1}(x)$. 
The convergence of the poles of $R_{n, m}(x)$ ( $m$ fixed) to those of $\Gamma(x)$ appears to be somewhat slow. This is probably due to the strength of the singularity of $\Gamma(x)$ at infinity. Apparently, low powers of $x$ are not sufficient to satisfactorily approximate this singularity, so that poles of $R_{n, m}(x)$ are needed on the right of the interval $[2,3]$ until relatively high powers of $x$ are available.

4. Acknowledgements. I would like to acknowledge the programming help of James A. Ayers for this work. He performed the difficult task of writing the program for carrying out the high precision calculations required for this study.

I would also like to acknowledge the use of the extremely versatile multiple precision interpretative program written by Alfred Beam at the National Bureau of Standards (he is now at the University of Maryland).

The values of $E_{10,0}^{\prime}$ and $E_{13,0}^{\prime}$ are taken from [1].

General Motors Corporation

Warren, Michigan

1. H. Werner \& R. Collinge, "Chebyshev approximations to the Gamma function," Math. Comp., v. 15, 1961, p. 195-197.

2. J. L. Walsh, Interpolation and Approximation, American Mathematical Society Colloq Publ. Vol. 20, 3rd edition, American Mathematical Society, Providence, Rhode Island, 1960.

3. J. L. WALSH, Private communication.

4. J. L. WALSH, "On approximation to an analytic function by rational functions of best approximation," Math. Z., v. 38, 1934, p. 163-176. 